\title{
The kinetics of mutarotation in L-fucose as monitored by dielectric and infrared spectroscopy
}

Wilhelm Kossack, Wycliffe Kiprop Kipnusu, Mateusz Dulski, Karolina Adrjanowicz, Olga Madejczyk, Ewa Kaminska, Emmanuel Urandu Mapesa, Martin Tress, Kamil Kaminski, and Friedrich Kremer

Citation: The Journal of Chemical Physics 140, 215101 (2014); doi: 10.1063/1.4880718

View online: https://doi.org/10.1063/1.4880718

View Table of Contents: http://aip.scitation.org/toc/jcp/140/21

Published by the American Institute of Physics

\section{Articles you may be interested in}

Mechanism of mutarotation in supercooled liquid phase: Studies on L-sorbose

The Journal of Chemical Physics 137, 124504 (2012); 10.1063/1.4752728

Kinetic processes in supercooled monosaccharides upon melting: Application of dielectric spectroscopy in the mutarotation studies of D-ribose

The Journal of Chemical Physics 132, 195104 (2010); 10.1063/1.3408286

Classification of secondary relaxation in glass-formers based on dynamic properties

The Journal of Chemical Physics 120, 857 (2004); 10.1063/1.1630295

Studies on mechanism of reaction and density behavior during anhydrous D-fructose mutarotation in the supercooled liquid state

The Journal of Chemical Physics 134, 175102 (2011); 10.1063/1.3575586

On the kinetics of tautomerism in drugs: New application of broadband dielectric spectroscopy

The Journal of Chemical Physics 133, 094507 (2010); 10.1063/1.3475688

Relaxation dynamics in plastic crystals

The Journal of Chemical Physics 116, 10386 (2002); 10.1063/1.1477186

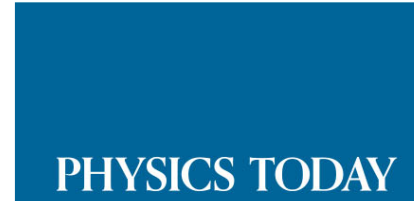

WHITEPAPERS
ADVANCED LIGHT CURE ADHESIVES

READ NOW

Take a closer look at what these environmentally friendly adhesive systems can do
PRESENTED BY

QMASTERBOND: 


\title{
The kinetics of mutarotation in L-fucose as monitored by dielectric and infrared spectroscopy
}

\author{
Wilhelm Kossack, ${ }^{1, a)}$ Wycliffe Kiprop Kipnusu, ${ }^{1}$ Mateusz Dulski, ${ }^{2}$ Karolina Adrjanowicz, ${ }^{3}$ \\ Olga Madejczyk, ${ }^{2}$ Ewa Kaminska, ${ }^{4}$ Emmanuel Urandu Mapesa, ${ }^{1}$ Martin Tress, ${ }^{1}$ \\ Kamil Kaminski, ${ }^{2}$ and Friedrich Kremer $^{1}$ \\ ${ }^{1}$ Institute of Experimental Physics, University of Leipzig, Linnestr. 5, 04103 Leipzig, Germany \\ ${ }^{2}$ Institute of Physics, University of Silesia, ul. Uniwersytecka 4, 40-007 Katowice, Poland and Silesian Center \\ of Education and Interdisciplinary Research, University of Silesia, 75 Pulku Piechoty 1A, 41-500 Chorzów, \\ Poland \\ ${ }^{3}$ NanoBioMedical Centre, ul. Umultowska 85, 61-614 Poznan, Poland \\ ${ }^{4}$ Department of Pharmacognosy and Phytochemistry, School of Pharmacy with the Division of Laboratory \\ Medicine in Sosnowiec, Medical University of Silesia in Katowice, ul. Jagiellonska 4, 41-200 Sosnowiec, \\ Poland
}

(Received 18 February 2014; accepted 19 May 2014; published online 6 June 2014)

\begin{abstract}
Fourier Transform Infrared Spectroscopy and Broadband Dielectric Spectroscopy are combined to trace kinetics of mutarotation in $L$-fucose. After quenching molten samples down to temperatures between $T=313 \mathrm{~K}$ and $328 \mathrm{~K}$, the concentrations of two anomeric species change according to a simple exponential time dependence, as seen by an increase in absorbance of specific IR-vibrations. In contrast, the dielectric spectra reveal a slowing down of the structural $(\alpha-)$ relaxation process according to a stretched exponential time dependence (stretching exponent of $1.5 \pm 0.2$ ). The rates of change in the IR absorption for $\alpha$ - and $\beta$-fucopyranose are (at $T=313 \mathrm{~K}$ ) nearly one decade faster than that of the intermolecular interactions as measured by the shift of the $\alpha$-relaxation. This reflects the fact that the $\alpha$-relaxation monitors the equilibration at a mesoscopic length scale, resulting from fluctuations in the anomeric composition. () 2014 AIP Publishing LLC. [http://dx.doi.org/10.1063/1.4880718]
\end{abstract}

\section{INTRODUCTION}

Saccharides are a huge family of compounds having numerous applications mainly in the pharmaceutical and food industry. Recent reports show that they can be used as energy supply in batteries or to stabilize the native structure of proteins or labile Active Pharmaceutical Ingredients. ${ }^{1-5}$ Carbohydrates also play a key role in many vital biophysical processes. ${ }^{6}$ These materials appear in a dynamic equilibrium of different isomeric states: i.e., in different cyclic or an open chain conformations. ${ }^{7-11}$ The interconversion between these states, termed mutarotation by Dubrunfaut in $1846,{ }^{12}$ is accompanied by a change of the specific optical rotation. ${ }^{8,13}$ Within the crystalline state, saccharides appear in only one of the forms shown in Fig. 1. ${ }^{14,15}$ But after melting or dissolving the crystallites, new isomers start to form. ${ }^{9,12,16}$ The kinetics of this reactions in solution and the populations of the given tautomers in their dependence on temperature, solvent, and other parameters have been studied before by polarimetry, ${ }^{8,9}$ nuclear magnetic resonance, ${ }^{10,11,17}$ chromatography, ${ }^{18-20}$ and quantum chemical calculations. ${ }^{21}$ For example, it was shown that the dielectric constant of the used solvent influences significantly the population of the anomers. ${ }^{8,20}$ In solution mutarotation is fast and it is not possible to eliminate effects of the solvent on the mechanism and speed of the reaction. And interestingly, recent studies revealed that in the molten state mutarotation behaves quite differently compared to its behavior in solution. ${ }^{15,22-27}$ In par-

\footnotetext{
a)Electronic mail: wilhelm.kossack@physik.uni-leipzig.de
}

ticular, in molten fructose the kinetic curves exhibit a sigmoidal shape and the activation barrier $\left(E_{\mathrm{a}} \sim 100 \mathrm{~kJ} / \mathrm{mol}\right)$ is almost twice as high as in solution. ${ }^{22}$ Furthermore, the activation energies of saccharides of different structure differ strongly (as in ribose: $:^{23} \sim 100 \mathrm{~kJ} / \mathrm{mol}$ and sorbose: ${ }^{24} \sim 60$ $\mathrm{kJ} / \mathrm{mol}$ ) proving the strong impact of structure and conformation on the kinetics of mutarotation. By means of Raman spectroscopy, Dujardin et al. recently investigated mutarotation of anomerically pure glucose obtained from mechanical milling. ${ }^{16,28}$ They report a kink in the temperature dependence of the rate constant of mutarotation occurring exactly at the calorimetric glass transition temperature. This further underlines the role of the overall structure and mobility on tautomerization.

Another interesting report was published by Tombari et al. showing that mutarotation can also take place in crystals. ${ }^{14}$ They rationalized that the energy of the molecules located close to lattice vacancies is high enough to trigger isomerization. As a result, the newly formed tautomers do not fit into the crystal geometry and instead create a liquid-like region that starts to spread. Consequently, liquefaction of crystals is observed well below the melting temperature.

In this paper, mutarotation kinetics of $L$-fucose is on the one hand monitored by broadband dielectric spectroscopy (BDS). This technique is sensitive to changes of the microscopic dipole moments in particular their concentration and mobility, ${ }^{29}$ and hence can be used to follow the kinetics of chemical reactions like the polymerization of resins and others. ${ }^{30-34}$ On the other hand, Fourier Transform Infrared 

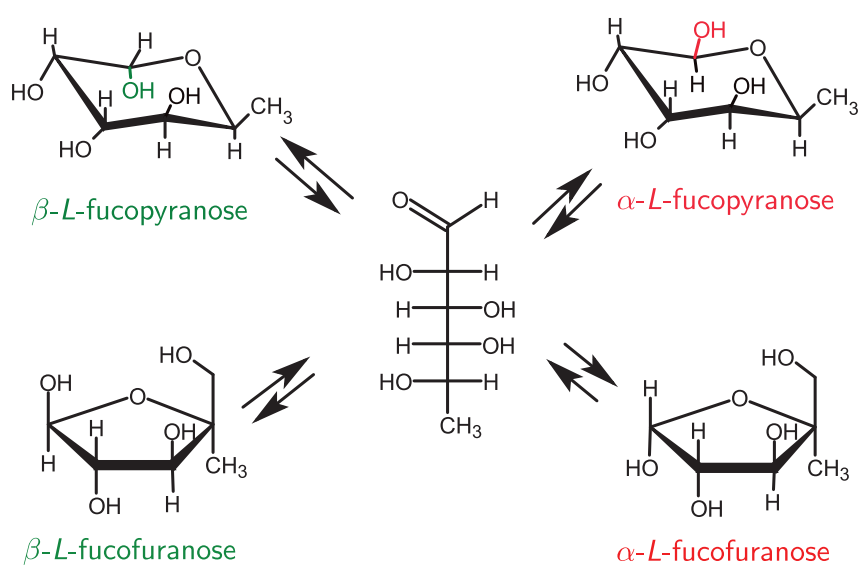

FIG. 1. Sketch of the various possible anomers of L-fucose. The linear conformation is a transitional state with negligible lifetime and concentration at any time scale of the experiments. ${ }^{7-10}$

spectroscopy (FTIR) is applied which specifically senses vibrational absorptions of different molecular moieties. ${ }^{35}$ By this combination the kinetics of mutarotation is traced on macroscopic and microscopic length scales, allowing deeper insights into the specific pathways of this reaction and its impact on macroscopic properties.

\section{EXPERIMENTAL}

Anhydrous $L$-fucose of high purity (99\%) was supplied by Sigma Aldrich. Its possible anomeric forms are depicted in Fig. 1 together with the generally accepted reaction path. ${ }^{7-9}$ For dielectric measurements (Alpha spectrometer Novo-Control $\mathrm{GmBH}$ ) the sugar powders are placed on a steel plate, then melted at $T=418 \mathrm{~K}$, capped by an upper electrode and quenched to ambient temperature. Then the sample is placed in a cryostat and the target temperature is stabilized (with $0.1 \mathrm{~K}$ accuracy) by the Quattro system (Novo-Control $\mathrm{GmBH})$ using dry nitrogen gas. Afterwards isothermal measurements were performed every $10 \mathrm{~min}$ in a frequency range of $f=1-10^{7} \mathrm{~Hz}$ (Fig. 2). For every temperature a new sample was prepared. The obtained spectra are fitted by HavriliakNegami-functions ${ }^{36}$ to obtain the relaxation times, $\tau_{\alpha}$, of the structural relaxation process. ${ }^{29}$

Samples for FTIR measurements are prepared in the same manner as described above but kept between $\mathrm{BaF}_{2}$ windows instead of metal electrodes. Spectra are collected at variable rates $(\lesssim 12 \mathrm{spectra} / \mathrm{min})$ at constant temperatures in the range of $700-6000 \mathrm{~cm}^{-1}$ and with a spectral resolution of $2 \mathrm{~cm}^{-1}$ using a Bio-Rad FTS-6000 spectrometer (Fig. 5). Temperature is controlled by a Linkam THMS 600 heating stage with an accuracy of about $0.1 \mathrm{~K}$. No indications of thermal or chemical degradation (caramelization) are observed in the FTIR spectra collected upon melting, nor do any color changes appear.

Additionally, $T$-dependent BDS data after equilibration for $12 \mathrm{~h}$ at $T=318 \mathrm{~K}$ are collected upon heating with a fast heating rate of $\approx 5 \mathrm{~K} / \mathrm{min}$. Also isothermal FTIR spectra at $333 \mathrm{~K}$ were collected 2 min after the kinetic measurement at $318 \mathrm{~K}$ had finished.

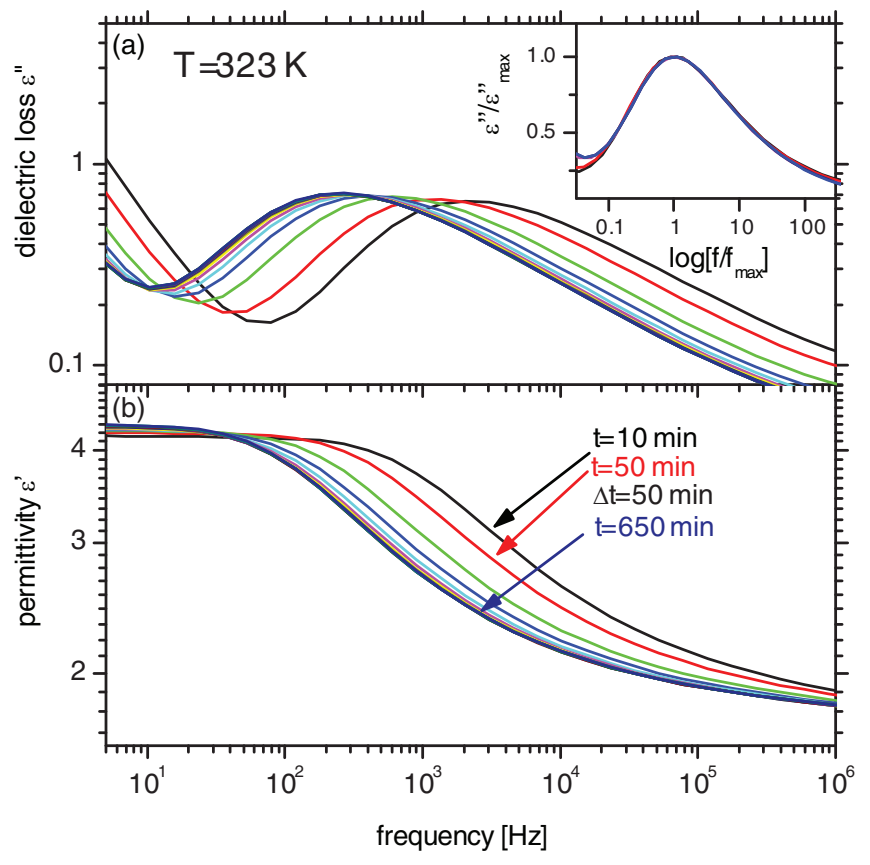

FIG. 2. Temporal evolution of dielectric spectra with time at $T=323 \mathrm{~K}$; (a) and (b), respectively, depict the imaginary and real part of the dielectric function: $\epsilon^{\prime}(f)-\mathrm{i} \epsilon^{\prime \prime}(f)$. (Inset) A time, annealing-time superposition of $\epsilon^{\prime \prime}$. Deviations at low frequency, $f$, are due to conductivity. Whereas at high $f$, distortions are caused by the decreased sensitivity of the spectrometer particularly for small mutarotation times.

\section{RESULTS AND DISCUSSION}

In Fig. 1 the interconversion between isomers due to mutarotation is sketched based upon a ring opening mechanism. ${ }^{7-9,26}$ These four tautomers differ in their dielectric properties, ${ }^{37}$ and hence mutarotation must influence the BDS data (Fig. 2). Within $12 \mathrm{~h}$ we observe a slowing down by more than one decade and a weak increase in dielectric strength $\left(\Delta \epsilon_{\alpha}\right)$ of the structural relaxation process. Effects on $\Delta \epsilon_{\alpha}$ and static permittivity (Fig. 2(b)), both increasing by about $2 \%$, are assigned to changes of the dipole-dipole correlation or to changes of the overall dipole moment, both probably evolving with $\tau_{\alpha}$. Additionally, one observes that the $d c$ conductivity shifts to lower frequencies. This may be explained by an increase of viscosity resulting in a reduced mobility of ionic impurities. ${ }^{26}$

We will focus here on the shift of $\tau_{\alpha}$ being most pronounced and well established. ${ }^{3,24,25,27}$ Such shifts could originate from different glass transition temperatures, $T_{\mathrm{g}}$, of the different anomeric fucopyranose and fucofuranose species, when their relative concentrations change. ${ }^{27}$ As the dipole moments of the fucose tautomers differ by up to a factor of three among each other, ${ }^{37}$ one would expect a change in shape and dielectric strength of the $\alpha$-relaxation in the course of concentration changes. But, that is not observed (Fig. 2). As fucose is a network forming liquid, the $\alpha$-relaxation originates rather from intermolecularly coupled reorientations of groups of molecules instead of rotations of the individual molecules. Therefore, the $\alpha$-peak cannot be considered as a simple sum of the relaxations of the four tautomers. More likely this process records the relaxation of a substructure of the H-bonding network, that must depend on the tautomeric 


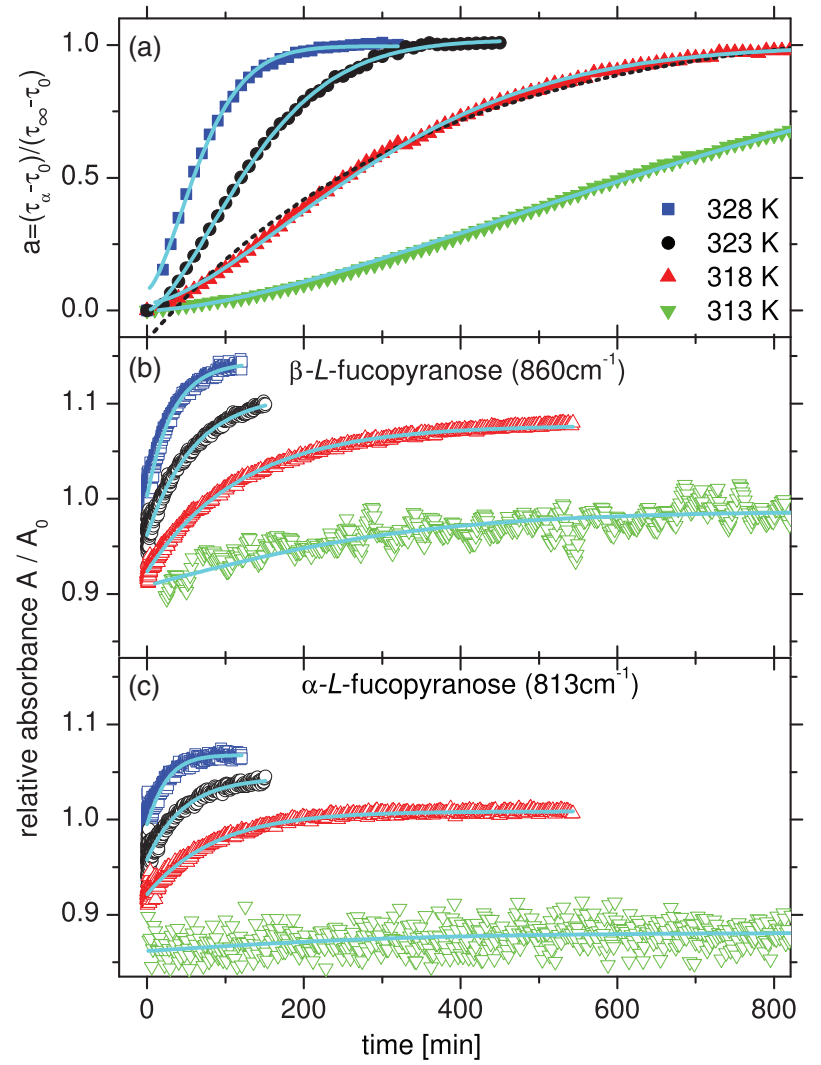

FIG. 3. Temporal evolution of $\tau_{\alpha}$ (a) being rescaled according to Eq. (1); integrated absorbance of vibration $v_{\beta}$ (b) and $v_{\alpha}$ (c). Cyan solid lines are fits according to Eq. (2) (see Table I). In (b) and (c) these fits resemble a single exponential growth. But in (a) a clearly sigmoidal shape $(n=1.5)$ is observed (the dashed line depicts an exponential fit with $n=1$ ). Dependencies in (b) and (c) are consecutively shifted downwards for lower temperatures to reduce overlap of the charts.

composition. This may very well explain the slowing down of that relaxation with an essentially unchanged shape and dielectric strength (Fig. 2). And furthermore it is in line with Tombari's interpretation of liquefaction of crystals below their melting point, which is based on structural and geometrical arguments. ${ }^{14}$ Finally, we need to mention that our measurements are performed well above $T_{\mathrm{g}}(305 \mathrm{~K})^{37}$ and therefore physical aging can be excluded as well as chemical degradation or crystallization, of which no indications are found in the BDS or FTIR data.

The time scales and amplitudes of the observed shift in $\tau_{\alpha}$ differ strongly between $328 \mathrm{~K}$ and $313 \mathrm{~K}$. And hence, the dielectric $\alpha$ relaxation times are presented in a normalized fashion in their dependence on reaction time, $t$,

$$
a=\frac{\tau_{\alpha}(t)-\tau_{0}}{\tau_{\infty}-\tau_{0}},
$$

where $\tau_{0}$ and $\tau_{\infty}$ are the initial and equilibrium relaxation times as deduced from fitting Eq. (2) to $\tau_{\alpha}(t)$. $a$ can be treated as a degree of conversion (Fig. 3(a)). At the lower temperatures a sigmoidal shape is recorded, which persists, although less pronounced, for higher temperatures $(T>318 \mathrm{~K})$ and has not been observed in solution. ${ }^{8,20}$ Similar stretched exponential kinetics of mutarotation indeed were reported in the vicinity of $T_{\mathrm{g}}$ for several pure mono-saccharides. ${ }^{24,27}$ To ex-
TABLE I. Parameters from modelling of the time dependencies by Eq. (2). For FTIR only the relative change of the integrated absorbance is displayed $\left(A_{\infty} / A_{0}\right)$, as the thickness of the samples is not exactly known. Absolute values for $\tau_{\infty}$ can also be estimated from Fig. 6. The Avrami exponent $n$ is 1.5 \pm 0.2 for BDS measurements and $1.0 \pm 0.1$ for FTIR.

\begin{tabular}{|c|c|c|c|c|}
\hline & $A_{\infty} / A_{0}(\%)$ & $\tau_{\infty}\left(10^{-4} \mathrm{~s}\right)$ & $\tau_{0}\left(10^{-4} \mathrm{~s}\right)$ & $k\left(10^{-4} \mathrm{~s}^{-1}\right)$ \\
\hline \multicolumn{5}{|c|}{$313 \mathrm{~K}$} \\
\hline$v_{\alpha}$ & $3.0 \pm 0.2$ & & & $1.1 \pm 0.5$ \\
\hline$v_{\beta}$ & $8.2 \pm 0.5$ & & & $0.65 \pm 0.06$ \\
\hline BDS & & $9400 \pm 400$ & $70 \pm 30$ & $0.18 \pm 0.02$ \\
\hline \multicolumn{5}{|c|}{$318 \mathrm{~K}$} \\
\hline$v_{\alpha}$ & $8.6 \pm 0.1$ & & & $1.9 \pm 0.1$ \\
\hline$v_{\beta}$ & $15.4 \pm 0.1$ & & & $1.4 \pm 0.1$ \\
\hline BDS & & $470 \pm 70$ & $9 \pm 4$ & $0.51 \pm 0.04$ \\
\hline \multicolumn{5}{|c|}{$323 \mathrm{~K}$} \\
\hline$v_{\alpha}$ & $8.6 \pm 0.1$ & & & $3.5 \pm 0.1$ \\
\hline$v_{\beta}$ & $14.9 \pm 0.1$ & & & $2.7 \pm 0.1$ \\
\hline BDS & & $33 \pm 3$ & $1.5 \pm 3$ & $1.06 \pm 0.03$ \\
\hline \multicolumn{5}{|l|}{$328 \mathrm{~K}$} \\
\hline$v_{\alpha}$ & $7.5 \pm 0.1$ & & & $6.5 \pm 0.2$ \\
\hline$v_{\beta}$ & $14.1 \pm 0.1$ & & & $5.0 \pm 0.1$ \\
\hline BDS & & $4 \pm 1$ & $0.5 \pm 1$ & $2.2 \pm 0.1$ \\
\hline \multicolumn{5}{|l|}{$333 \mathrm{~K}$} \\
\hline$v_{\alpha}$ & $-3.4 \pm 0.6$ & & & $10.69 \pm 0.8$ \\
\hline$v_{\beta}$ & $-2.2 \pm 0.4$ & & & $8 \pm 3$ \\
\hline
\end{tabular}

tract rate constants, $k_{\mathrm{BDS}}$, considering the sigmoidal shape, a renormalized Avrami model is used to describe $\tau_{\alpha}(t),{ }^{38}$

$$
\tau_{\alpha}(t)=\tau_{0}+\left(\tau_{\infty}-\tau_{0}\right) \exp \left[-(k t)^{n}\right]
$$

where $n$ is the Avrami exponent. As can be seen in Fig. 3(a), this approach describes the experimental data very well, and furthermore, it yields the same constant rates as it would without renormalization. Small systematic differences of the resulting fit parameters are found when compared to the results of Wlodarczyk et al. and are assigned to the usage of a different fitting function (Table I). ${ }^{27}$ Using $n$ as a shared parameter for all four $\tau_{\alpha}(t)$ charts we obtain $n=1.5 \pm 0.2$. When Eq. (2) is used to describe concentration changes directly, $n$ can be correlated with the mechanism of reaction. ${ }^{39}$ But here a dynamic quantity is modeled, which is strongly dependent on intermolecular interactions and all attempts to assign a physical meaning to such an $n$ are still controversially discussed. $^{40-42}$

To gain deeper insight into the mechanism of mutarotation in $L$-fucose, FTIR was applied. We calculated theoretical FTIR spectra for each cyclic isomer (Fig. 1) using density functional theory (DFT), ${ }^{37,43}$ and compared them to spectra of the crystalline, partially crystalline and amorphous state of $L$-fucose as well as literature data of glucose (Fig. 4). ${ }^{16} \mathrm{By}$ that, we assigned absorption bands at $\sim 813 \mathrm{~cm}^{-1}\left(v_{\alpha}\right)$ and $\sim 860 \mathrm{~cm}^{-1}\left(v_{\beta}\right)$ to ring vibrations of the $\alpha$ and $\beta$ form of fucopyranose, respectively. From the presence of the vibrational bands indicated in Fig. 4, it can be concluded that only $\alpha$-fucopyranose anomers are present in the initial crystalline state, whereas the amorphous state at least exhibits the $\beta$ fucopyranose forms as well. We have chosen $v_{\alpha}$ and $v_{\beta}$ to 


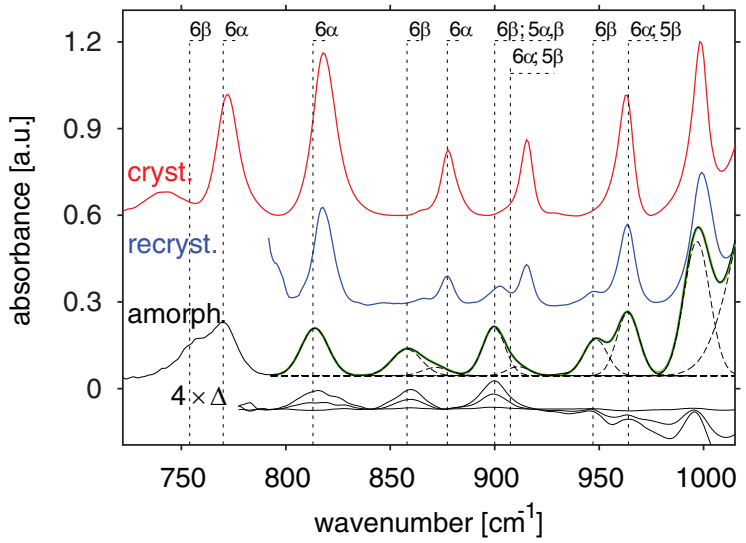

FIG. 4. Comparison of FTIR spectra in the low $v$ region of $L$-fucose at ambient temperatures from top downwards: crystalline state (as received, red), recrystallized after melting with some amorphous content (blue), and in the amorphous state (black, solid). For the last spectrum the deconvolution into Gaussian peaks (black dashed) is shown together with the cumulative fit (thick green line). The lowest 3 charts ( $\Delta$, thinner black lines) are magnified difference spectra $(4 \times)$, illustrating the temporal evolution of $A(v)$ at $323 \mathrm{~K}$. Vertical dashed lines depict vibrational frequencies as revealed from DFT calculations for the different tautomers: fucopyranose (denoted as 6) and -furanose (5) in the $\alpha$ or $\beta$ state. All charts are shifted vertically for clarity.

follow the rate of formation of the corresponding isomers, as they can be clearly identified and do not have significant overlap with bands of uncertain assignment. Furthermore, it is unfortunately impossible in the accessible frequency range to assign IR active vibrations specifically to each one of the four fucose forms (Figs. 1 and 4). Hence, the extraction of rates for $\alpha$ - and $\beta$-L-fucofuranose was not possible. Here we assume implicitly that the oscillator strength of the two discussed bands is independent of concentration, local structure and measurement time, which of course is an approximation.

In Fig. 5 representative FTIR spectra obtained during annealing of $L$-fucose at $323 \mathrm{~K}$ for $13 \mathrm{~h}$ are presented together with the corresponding FTIR difference spectra with respect to the initial state, $\Delta$. The two aforementioned bands $v_{\alpha}$ and $v_{\beta}$ are analyzed further by fitting Gaussian functions reveal-

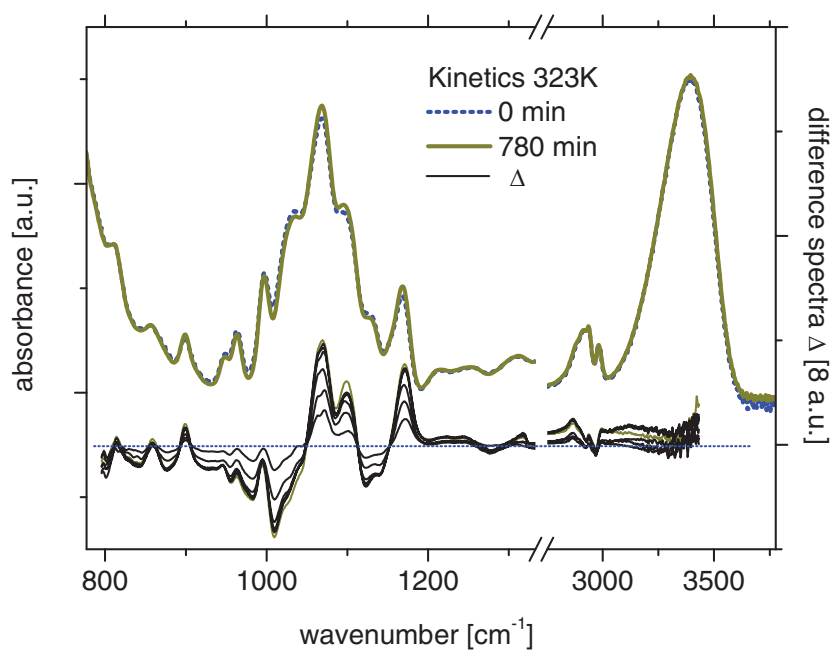

FIG. 5. Evolution of IR spectra with time at $T=323 \mathrm{~K}$, The lower region (thinner lines) depicts difference spectra, $\Delta$, with respect to the first spectrum. These refer to the right $y$ scale being expanded by a factor of 8 . ing integrated absorbance $(A)$, width and spectral position (Fig. 4). Interestingly, the latter two quantities do not change within measurement time, proving that these bands originate from an unchanged vibrational energy landscape. On the other hand, for $318 \mathrm{~K}<T<328 \mathrm{~K}, A$ increases as the reaction proceeds by $(8 \pm 2) \%$ for $v_{\alpha}$ and by $(15 \pm 3) \%$ for $v_{\beta}$ when compared to $A_{0}=A(t=0)$ (Figs. 3(b) and 3(c)). That increase becomes much slower and a little weaker as temperature is lowered and, consequently, at $T=313 \mathrm{~K}$ the data are quite scattered. According to a first, rough approximation, we can identify the ratio $A / A_{0}$ with the change in concentration of the corresponding tautomer. And hence, we conclude that the rate of formation of $\alpha$ - and $\beta$-fucopyranose is greater at higher temperatures and that the significant slowing down of the structural relaxation process as revealed by dielectric measurements is connected to the growing number of pyranose isomers (Fig. 2). The simultaneous increase in $\Delta \epsilon_{\alpha}$, although the dipole moment of pyranose- is smaller than the one of furanose-forms, ${ }^{37}$ corroborates the assignment of the $\alpha$-process as a relaxation of a substructure of the hydrogen bonding network. Unfortunately, the deduction of absolute or even relative concentrations from $A$ is complicated by the following: (i) The ratio of the oscillator strength of the discussed bands depending on temperature is not known; and furthermore can not assumed to be constant, as we do not find a linear correlation of them. (ii) To the best of our knowledge there is no temperature at which the individual concentrations of the tautomers are known. (iii) The oscillator strength must depend on the microscopic structure, that changes drastically upon mutarotation, as can be seen from the shift of $\tau_{\alpha}$.

Modelling $A(t)$ with Eq. (2) (Figs. 3(b) and 3(c) and Table I) reveals constant rates of production, $k_{\mathrm{IR}, \alpha / \beta}$, for $\alpha$ $\left(v_{\alpha}\right)$ and $\beta$-fucopyranose $\left(v_{\beta}\right)$. The rate $k_{\mathrm{IR}, \alpha}$ is at $T=313 \mathrm{~K} \mathrm{a}$ factor of $\sim 1.7$ faster than $k_{\mathrm{IR}, \beta}$, but their difference decreases for higher temperatures (Fig. 7). Nevertheless, both are significantly faster than $k_{\mathrm{BDS}}$. Interestingly, $A(t)$ of each band follows a simple single exponential trend $(n=1 \pm 0.1)$ indicating that FTIR measurements indeed reveal a change in concentration of the various anomers, whereas the $\alpha$-relaxation records more macroscopic and cooperative effects (Fig. 3). Furthermore, the data do not indicate a preceding fast conversion step as seen for fructose in viscosity measurements and assigned to a fast conformational equilibration by Wang et al., although FTIR is sensitive to such changes. ${ }^{26}$

Raising the temperature again to $333 \mathrm{~K}$ after mutarotation has equilibrated at $318 \mathrm{~K}$ inverts the effects on the FTIR spectra, as one would expect. But as the temperature step $(+15 \mathrm{~K})$ and therefore the change in the equilibrium concentrations are small: $A / A_{0}$ decreases only by $(2.5 \pm 1) \%$ upon time for $v_{\alpha}$ and $v_{\beta}$. Due to that, the corresponding rates have a large uncertainty, and hence further rates are presented in Fig. 7 corresponding to vibrations at $\sim 900 \mathrm{~cm}^{-1}$ and $\sim 946 \mathrm{~cm}^{-1}$ (Fig. 4). These bands show a change in $A / A_{0}$ above the signal to noise ratio and corroborate the time scales extracted from $v_{\alpha}$ and $v_{\beta}$. Interestingly, the $k_{\mathrm{IR}}$ 's extracted from such a measurement with $\Delta T>0$ perfectly match up with the rates from experiments upon lowering $T$. This indicates that the same mechanism governs both directions of the reaction (data at $1000 / T \approx 3 \mathrm{~K}^{-1}$ in Fig. 7 refer to $\Delta T=+15 \mathrm{~K}$ ). 


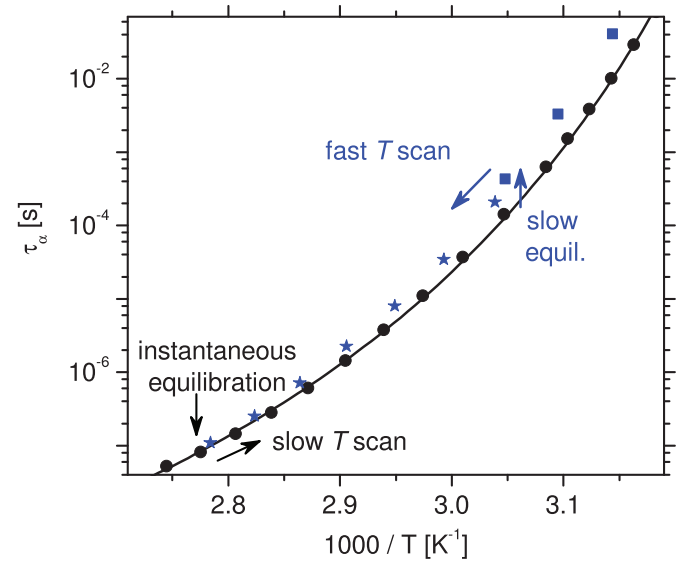

FIG. 6. Structural relaxation time vs. $1000 / T, \tau_{\alpha}$ obtained from the equilibrium state of the mutarotation experiments are depicted as blue squares. Blue stars represent the characteristic times extracted from fast $(5 \mathrm{~K} / \mathrm{min})$ subsequent heating after mutarotational equilibrium at $T=318 \mathrm{~K}$ was reached (slow equil. $\tau \gtrsim 10^{3} \mathrm{~s}$ ). Black circles refer to the standard (slow $1 \mathrm{~K} / \mathrm{min}$ ) $T$ scan measurement after equilibration at $418 \mathrm{~K}$ and the black line shows the corresponding Vogel-Fulcher-Tamman fit (data taken from Ref. 37).

Inspired by that, we recorded $\tau_{\alpha}$ after equilibration at $318 \mathrm{~K}$ upon fast heating $(\approx 5 \mathrm{~K} / \mathrm{min})$ and compare the results to the $\alpha$-relaxation rates obtained upon slow cooling $\left(\approx 1 \mathrm{~K} / \mathrm{min}\right.$ ) from $T=418 \mathrm{~K}$ (Fig. 6). ${ }^{37}$ The two $\tau_{\alpha}$ 's merge around $T_{\text {eq }}=(355 \pm 5) \mathrm{K} \approx T_{\mathrm{g}} / 0.86$, meaning the concentrational equilibration is reached within the experimental time scale $\tau_{\exp } \sim 1 \mathrm{~min}$. At this temperature the rates of mutarotation, extrapolated to high $T$ by Eq. (3), roughly agree with each other: $k_{\mathrm{IR} \alpha / \beta} \approx k_{\mathrm{BDS}} \sim 1 \mathrm{~min}^{-1}$. That agreement of $k$, also matching $\tau_{\mathrm{exp}}^{-1}$, corroborates the assumption of an Arrhenius-like $T$-dependence from 313 up to $T_{\text {eq }} \approx 355 \mathrm{~K}$. Interestingly, above $T_{\text {eq }}$ we find the merging of the structural$(\alpha-)$ and a secondary relaxation in the BDS spectra, which has been interpreted as a weakening of dynamic barriers between inter-molecular, structural substates in contrast to only

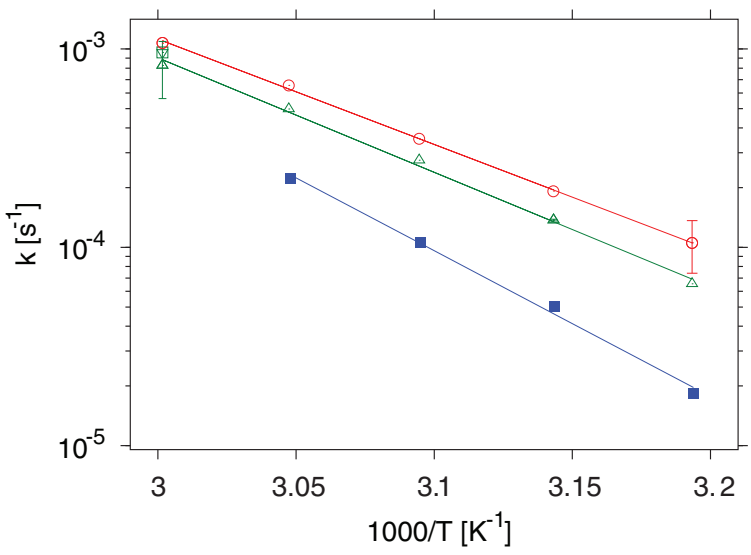

FIG. 7. Rates, $k$ (as extracted from fitting Eq. (2)), vs. inverse temperature, Open symbols depict rates extracted from FTIR measurements-red circles: $\alpha$-fucopyranose $\left(v_{\alpha}\right)$, green upwards triangles: $\beta$-fucopyranose $\left(v_{\beta}\right)$, open square $\left(900 \mathrm{~cm}^{-1}\right)$, and downwards triangle $\left(946 \mathrm{~cm}^{-1}\right)$ may originate from multiple species (see Fig. 4). The latter two symbols only appearing at 1000/T $=3 \mathrm{~K}^{-1}(333 \mathrm{~K})$ correspond to a jump in temperature from $318 \mathrm{~K}$ to $333 \mathrm{~K}$, whereas all others are obtained after a quench from $418 \mathrm{~K}$. Filled symbols refer to $\tau_{\alpha}$ from BDS. The solid lines represent fits of Eq. (3) to $k$. Error bars are drawn, if not smaller than the symbol-size. weakly influenced intra-molecular dynamics. ${ }^{44}$ Hence, the time-dependencies of mutrotation and particularly the differences between FTIR and BDS are expected to change qualitatively here (especially for the Avrami exponent: $n_{\mathrm{BDS}} \rightarrow 1$ ). Therefore, studies of the dynamics of mutarotation around and above $T_{\mathrm{eq}}$ on the second to minute time scale would be very welcome, but cannot be performed with our equipment.

Finally, the activation barrier, $E_{\mathrm{a}}$, of mutarotation in $L$ fucose is extracted by fitting the Arrhenius equation (Eq. (3)) to the different constant rates obtained (see Fig. 7), again assuming a constant relation of oscillator strength and concentration,

$$
\log _{10} k(T)=\log _{10} k_{0}-\frac{E_{\mathrm{a}}}{k_{\mathrm{B}} T},
$$

where $k_{0}$ is a pre-exponential factor and $k_{\mathrm{B}}$ the Boltzmann constant. We obtain $E_{\mathrm{a}} \approx 139 \pm 7 \mathrm{~kJ} / \mathrm{mol}$ from $k_{\mathrm{BDS}}{ }^{45}$ The respective $E_{\mathrm{a}}(\mathrm{IR})$ for $v_{\alpha}$ and $v_{\beta}$ is determined to be 102 and $111 \mathrm{~kJ} / \mathrm{mol}$, with a similar uncertainty as $E_{\mathrm{a}}(\mathrm{BDS})$. The fact that $E_{\mathrm{a}}(\mathrm{IR})$ is roughly $18 \%-28 \%$ smaller than the one obtained from BDS agrees with the assignment of the $\alpha$ relaxation to a super-molecular aggregate.

\section{CONCLUSION}

FTIR and BDS are employed to study mutarotation in molten $L$-fucose between 313 and $333 \mathrm{~K}$. Both techniques provide complementary information and give insights into the interconversion of the different anomers. Upon reaction time FTIR spectra show an increasing absorbance for specific IR vibrations of $\alpha$ - and $\beta$-fucopyranose-anomers following first order kinetics (single exponential). From that, the relative concentration of the corresponding anomers is deduced to be about $8 \%$ (for $\alpha$-fucopyranose) and 15\% ( $\beta$-fucopyranose) higher than at $T_{\mathrm{eq}}=355 \mathrm{~K}$, assuming temperature independent oscillator strength. This change of the composition causes a slowing down of the dielectric $\alpha$-relaxation having a stretched exponential time dependence (exponent: $1.5 \pm 0.2$ ). The characteristic rates, $k$, as deduced from BDS are, especially for the lower temperatures, significantly smaller than the ones obtained from FTIR. All $k_{\mathrm{IR}}$ 's and $k_{\mathrm{BDS}}$ follow Arrhenius like temperature dependencies. The activation energies differ between BDS $\left(E_{a}=139 \mathrm{~kJ} / \mathrm{mol}\right)$ and FTIR, where values specific for $\alpha$-fucopyranose $\left(E_{a}=102 \mathrm{~kJ} / \mathrm{mol}\right)$ and $\beta$ fucopyranose $\left(E_{a}=111 \mathrm{~kJ} / \mathrm{mol}\right)$ are found. However, it is not possible to monitor the formation of fucofuranose moieties or determine absolute concentrations depending on temperature, as no FTIR bands specific for all isomeric species could be identified.

\section{ACKNOWLEDGMENTS}

The authors thank "Build-Mona-the Leipzig School of Natural Science"; Sächsische Forschgruppe: "FOR 877 From Local Constraints to Macroscopic Transport" and the Deutsche Forschungsgemeinschaft (within the SFB-TRR 102 and SPP 1369) for financial support. K.K. furthermore acknowledges financial assistance from the National Center of Science based on decision DEC-2012/05/D/ST4/00326. E.K. 
is thankful for the support from the National Center of Science based on decision DEC-2013/09/D/NZ7/04194. O.M. is grateful for the financial support from the Polish National Science Centre within the program OPUS3 (decision No. DEC2012/05/B/ST3/02837). This research was also supported in part by PL-Grid Infrastructure.

${ }^{1}$ T. Irie and K. Uekama, J. Pharm. Sci. 86, 147 (1997).

${ }^{2}$ K. Grzybowska, M. Paluch, P. Wlodarczyk, A. Grzybowski, K. Kaminski, L. Hawelek, D. Zakowiecki, A. Kasprzycka, and I. Jankowska-Sumara, Mol. Pharmaceutics 9, 894 (2012).

${ }^{3}$ B. Wang, S. Tchessalov, M. T. Cicerone, N. W. Warne, and M. J. Pikal, J. Pharm. Sci. 98, 3145 (2009).

${ }^{4}$ J. F. Carpenter, M. J. Pikal, B. S. Chang, and T. W. Randolph, Pharm. Res. 14, 969 (1997).

${ }^{5}$ E. Kaminska, K. Adrjanowicz, K. Kaminski, P. Wlodarczyk, L. Hawelek, K. Kolodziejczyk, M. Tarnacka, D. Zakowiecki, I. Kaczmarczyk-Sedlak, J. Pilch, and M. Paluch, Mol. Pharmaceutics 10, 1824 (2013).

${ }^{6}$ D. J. Becker and J. B. Lowe, Glycobiology 13, 41R (2003).

${ }^{7}$ V. A. Yaylayan, A. A. Ismail, and S. Mandeville, Carbohydr. Res. 248, 355 (1993).

${ }^{8}$ R. S. Shallenberger, Pure Appl. Chem. 50, 1409 (1978).

${ }^{9}$ M. Cockman, D. G. Kubler, A. S. Oswald, and L. Wilson, J. Carbohydr. Chem. 6, 181 (1987).

${ }^{10}$ D. Horton and Z. Wałaszek, Carbohydr. Res. 105, 145 (1982).

${ }^{11}$ C. Williams and A. Allerhand, Carbohydr. Res. 56, 173 (1977).

${ }^{12}$ A. P. Dubrunfaut, C. R. Acad. Sci. 23, 38 (1846).

${ }^{13}$ F. A. Carey, Organic Chemistry, 4th ed. (McGraw-Hill, Boston, 2000).

${ }^{14}$ E. Tombari, C. Ferrari, G. Salvetti, and G. P. Johari, J. Chem. Phys. 126, 021107 (2007).

${ }^{15}$ N. Dujardin, J. F. Willart, E. Dudognon, F. Danède, and M. Descamps, J. Phys. Chem. B 117, 1437 (2013).

${ }^{16}$ N. Dujardin, J. Willart, E. Dudognon, A. Hédoux, Y. Guinet, L. Paccou, B. Chazallon, and M. Descamps, Solid State Commun. 148, 78 (2008).

${ }^{17}$ S. J. Angyal and V. A. Pickles, Carbohydr. Res. 4, 269 (1967).

${ }^{18}$ D. Schumacher and L. W. Kroh, Food Chem. 54, 353 (1995).

${ }^{19}$ N. Le Barc'H, J. Grossel, P. Looten, and M. Mathlouthi, Food Chem. 74, 119 (2001).

${ }^{20}$ A. E. Flood, M. R. Johns, and E. T. White, Carbohydr. Res. 288, 45 (1996).

${ }^{21}$ A. M. Silva, E. C. da Silva, and C. O. da Silva, Carbohydr. Res. 341, 1029 (2006).

${ }^{22} \mathrm{P}$. Wlodarczyk, K. Kaminski, K. Adrjanowicz, Z. Wojnarowska, B. Czarnota, M. Paluch, J. Ziolo, and J. Pilch, J. Chem. Phys. 131, 125103 (2009).
${ }^{23}$ P. Wlodarczyk, K. Kaminski, S. Haracz, M. Dulski, M. Paluch, J. Ziolo, and M. Wygledowska-Kania, J. Chem. Phys. 132, 195104 (2010).

${ }^{24}$ P. Wlodarczyk, M. Paluch, A. Grzybowski, K. Kaminski, A. Cecotka, J. Ziolo, and J. Markowski, J. Chem. Phys. 137, 124504 (2012).

${ }^{25}$ P. Wlodarczyk, M. Paluch, L. Hawelek, K. Kaminski, and J. Pionteck, J. Chem. Phys. 134, 175102 (2011).

${ }^{26}$ Y. Wang, P. Wlodarczyk, A. P. Sokolov, and M. Paluch, J. Phys. Chem. B 117, 1475 (2013).

${ }^{27}$ P. Wlodarczyk, A. Cecotka, K. Adrjanowicz, K. Kaminski, and M. Paluch, J. Phys.: Condens. Matter 25, 375101 (2013).

${ }^{28}$ N. Dujardin, E. Dudognon, J.-F. Willart, A. Hédoux, Y. Guinet, L. Paccou, and M. Descamps, J. Phys. Chem. B 115, 1698 (2011).

${ }^{29}$ F. Kremer and A. Schönhals, Broadband Dielectric Spectroscopy (Springer, 2003).

${ }^{30}$ R. Casalini, S. Corezzi, A. Livi, G. Levita, and P. A. Rolla, J. Appl. Polym. Sci. 65, 17 (1997).

${ }^{31}$ G. P. Johari, J. G. McAnanama, and D. A. Wasylyshyn, J. Chem. Phys. 105, 10621 (1996).

${ }^{32}$ B. D. Fitz and J. Mijovic, J. Phys. Chem. B 104, 12215 (2000).

${ }^{33}$ Z. Wojnarowska, P. Wlodarczyk, K. Kaminski, K. Grzybowska, L. Hawelek, and M. Paluch, J. Chem. Phys. 133, 094507 (2010).

${ }^{34}$ Z. Wojnarowska, M. Paluch, P. Wlodarczyk, M. Dulski, R. Wrzalik, and C. M. Roland, J. Phys. Chem. Lett. 3, 2288 (2012).

${ }^{35} \mathrm{P}$. Griffiths and J. A. d. Haseth, Fourier Transform Infrared Spectrometry, 2nd ed. (Wiley-Interscience, 2007).

${ }^{36}$ S. Havriliak and S. Negami, J. Polym. Sci., Part C: Polym. Symp. 14, 99117 (1966).

${ }^{37}$ W. Kossack, K. Adrjanowicz, M. Tarnacka, W. K. Kipnusu, M. Dulski, E. U. Mapesa, K. Kaminski, S. Pawlus, M. Paluch, and F. Kremer, Phys. Chem. Chem. Phys. 15, 20641 (2013).

${ }^{38}$ M. Avrami, J. Chem. Phys. 9, 177 (1941).

${ }^{39}$ M. C. Weinberg, D. P. Birnie III, and V. A. Shneidman, J. Non-Cryst. Solids 219, 89 (1997).

${ }^{40}$ R. L. Leheny and S. R. Nagel, Phys. Rev. B 57, 5154 (1998).

${ }^{41}$ P. Lunkenheimer, R. Wehn, U. Schneider, and A. Loidl, Phys. Rev. Lett. 95, 055702 (2005)

${ }^{42}$ P. Lunkenheimer, R. Wehn, and A. Loidl, J. Non-Cryst. Solids 352, 4941 (2006).

${ }^{43}$ M. J. Frisch, G. W. Trucks, H. B. Schlegel et al., Gaussian 09, Revision A.1, Gaussian Inc., Wallingford, CT, 2009.

${ }^{44} \mathrm{At} T_{\text {eq }}$ several effects are observed in the $T$-dependent BDS and FTIR data. The interested reader is referred to the molecular dynamics section of Ref. 37.

${ }^{45}$ This activation energy roughly agrees with $E_{\mathrm{a}}=124 \pm 9 \mathrm{~kJ} / \mathrm{mol}$, as estimated earlier by some of us. ${ }^{27}$ 